\title{
Comparison between two diagnostic system such analyzer I and RAD I 20 for the determination of IgG and IgM for HSV-I and HSV-2
}

\author{
Annelisa Borelli', Vincenzina Caruso', Maria Teresa Cerminara', Salvatore Nisticò', Vilma Villella', \\ Pasquale Minchella², Rosa Anna Leone' \\ I SOC Microbiologia e Virologia , P.O. di Lamezia Terme, Azienda Sanitaria Provinciale di Catanzaro \\ 2 SOC Laboratorio Analisi Chimica-Clinica-RIA, Azienda Ospedaliera "Pugliese-Ciaccio", Catanzaro
}

Key Words: Herpes Simplex Virus, IgG, IgM, Antibody

Comparazione dei risultati ottenuti con Analyzer I e RAD I 20 nella diagnostica sierologica delle infezioni da Herpes Simplex Virus di tipo I e di tipo 2

\section{SUMMARY}

Herpes Simplex Virus (Herpesviridae family, Alphaherpesviridae subfamily) induces latent infections, which could reactivate in conjunction with decreases in cell-mediated response. Features biologic and antigenic of Herpes Simplex virus are characterized from HSV-I e HSV-2. Seronegative women can contract primary infection from seropositive partners. Seronegative women may acquire primary infection from an infected partner. Prevention is done paying attention to the risk of HSV2 in the planning and gravidnza pportune neonatal infection prevention measures (clinical examination of the birth canal at the beginning of labor). Disease prevention is performed by planning preventive measures to avoid neonatal infection. Infection occurs through direct contact with herpetic lesions or biological fluid infect. HSV-I is usually acquired about 5 years, whether HSV-2 is contracted between 14 and 30 years by sexual contact, both can be asymptomatic and be transmitted to the partner or to the newborn during the delivery through the contact with infected secretion.

The baby infected $85 \%$ of cases acquired the infection to step into the birth canal, through contact with infected secretions.

The intrauterine infection is proven in $5-8 \%$ of cases; in the $8-10 \%$ of cases postnatal infection the disease spreading occurs through breast milk or herpes skin lesions. The assessment of immune status can be evaluated by different serological methods, for determinate the presence of IgG and / or IgM anti-HSV-I and anti-HSV-2. Aim of this work is the comparison between two diagnostic systems such Analyzer I (Euroimmun) and RAD I20 (Radim) for the determination of IgG and IgM anti-HSV-I and anti-HSV-2. Between July 2009 - December 2009 n. I 82 samples have been tested with Analyzer I, automated ELISA system automatic, and system Rad I20, final fluorescent determination. From the obtained results we conclude that both the methods perform quit well.

\section{INTRODUZIONE}

Il Virus Herpes Simplex (HSV), virus a DNA a doppio filamento con simmetria icosaedrica, appartenente alla famiglia herpesviridae, sottofamiglia alpha-herpesviridae, è caratterizzato da un ciclo replicativo piuttosto breve e dalla capacità di infettare cellule epiteliali, indurre un marcato effetto citopatico, presentare uno spiccato neurotropismo e rimanere in stato latente nei gangli nervosi sensitivi (5); provoca generalmente infezioni non gravi che, nella maggior parte dei casi, si risolvono senza ricorso a terapia. Durante la gravidanza, tuttavia, HSV può infettare sia la madre che il feto, con conseguenze a volte gravi. Si distinguono due tipi di HSV, denominati rispettivamente tipo 1 (HSV-1) e tipo 2 (HSV-2), geneticamente differenti, ma strettamente correlati, responsabili di infezioni localizzate più frequentemente in due distretti: oro-labiale e genitale (4).

Tutte le manifestazioni cliniche oro-labiali sono correlate ad HSV-1, quelle genitali possono essere correlate ad entrambi i tipi. È difficile documentare, con i soli dati sierologici, quante forme cliniche genitali siano da attribuire ad HSV-1, tuttavia, è stato segnalato un recente significativo aumento di infezioni genitali da HSV-1 (3). Entrambe le infezioni possono decorrere in modo asintomatico ed essere quindi trasmesse al partner o, nel caso di donna in gravidanza, al bambino durante il passaggio nel canale del parto. L'infezione contratta in gravidanza o la riattivazione di un'infezione precedentemente acquisita può indurre nel neonato un'infezione congenita o neonatale; la severità del quadro clinico è correlata allo stato immunologico materno, al grado di replicazione ed escrezione virale ed alle modalità del parto (1).

La prevenzione dell'infezione neonatale da HSV è effettuata ponendo attenzione al rischio di infezione erpetica genitale in gravidanza praticando, ad esempio, un accurato esame clinico del canale del parto e della zona vulvare all’inizio del tra- vaglio e ricorrendo, nel caso di lesioni erpetiche, al parto cesareo come misura preventiva (2).

Le donne prive di titolo anticorpale anti-HSV (sieronegative) hanno un alto rischio di acquisire l'infezione primaria da un partner sieropositivo; è importante quindi eseguire test sierologici per HSV-1 e HSV-2 sia nella donna che nel partner per conoscere lo stato immunitario e, nel caso di sieronegatività della gravida, prendere opportune misure preventive. La valutazione dello stato immunitario nei confronti di HSV può essere effettuata mediante differenti metodi sierologici, che rivelano la presenza di anticorpi IgG e/o IgM.

Lo scopo di questo lavoro è la comparazione della performance di due strumenti e dei relativi reagenti utilizzati per determinare gli anticorpi IgG e IgM anti-HSV-1 ed anti-HSV-2.

\section{MATERIALI E METODI}

Sono stati testati, nel periodo luglio - dicembre 2009, tutti i campioni ematici (n. 182) provenienti da pazienti con richiesta di anticorpi anti-HSV-1 ed anti-HSV-2, sia ricoverati in vari Reparti di degenza del P.O. di Lamezia Terme (ASP di Catanzaro), sia utenti esterni afferenti ai diversi Centri prelievi territoriali. La maggior parte dei campioni testati era prelevato da donne in gravidanza sottoposte a screening o a monitoraggio sierologico, il resto da pazienti ricoverati con sintomatologia suggestiva di infezione erpetica.

È mtato eseguito il test, in uso di routine nell'Unità Operativa, mediante l'apparecchio Analyzer I (7), Ditta Euroimmun AG, Luebeck, Germania, quale test di riferimento. Analyzer I è un sistema aperto e completamente automatico per saggi in ELISA, in grado di processare fino a sette piastre da 96 pozzetti ed effettuare più di 12 differenti test direttamente da provetta primaria. I reagenti ed i controlli, specifici per ogni analita e pronti all'uso, sono alloggiati in appositi racks e vengono riconosciuti per lettura di barcode.

\section{Corresponding author: Annelisa Borelli}

SOC Microbiologia e Virologia, Presidio Ospedaliero di Lamezia Terme

Via A. Perugini I, 88046 Lamezia Terme - CZ - Tel.: 096820876 I - Fax:0968463 I I3

E-mail: lisi.borelli@libero.it 
Successivamente è stato effettuato il test mediante lo strumento RAD 120, Ditta Radim Diagnostics, Roma, su sieri conservati al massimo per 7 giorni alla temperatura di $-20^{\circ} \mathrm{C}$. Il sistema RAD 120 permette l'automazione di dosaggi basati su metodica fluoroimmunometrica mediante l'utilizzo della tecnologia brevettata "PEGASUS", in cui la fase solida è costituita da particelle magnetiche rivestite di zirconia. La rivelazione della fluorescenza è basata sull'uso di coniugati marcati con l'enzima fosfatasi alcalina e del substrato 4-metilumbelliferilfosfato (4-MUP). La fluorescenza ottenuta per ciascun campione viene interpolata sulla curva dose-risposta (curva di lavoro), relativa a ciascun analita. I reagenti, liquidi pronti all'uso, sono alloggiati in apposite cartucce su uno speciale contenitore rotante e possono rimanere a bordo poiché il vano è refrigerato a $2-8^{\circ} \mathrm{C}$.

Per la metodica Euroimmun è prevista la prediluizione del siero mediante un diluente già dotato di sorbent per la rimozione del fattore Reumatoide, mentre per l'esecuzione della metodica Radim non è previsto alcun assorbimento. La metodica Euroimmun prevede la ricerca separata di HSV-1 IgM e HSV-2 IgM, mentre la metodica Radim rivela HSV-1/2 IgM, non differenziandole per tipo.

I campioni risultati discordanti con i due sistemi, sono stati testati mediante due ulteriori sistemi in uso nell'Unità Operativa per la verifica dei casi dubbi: lo strumento Chorus, Ditta Diesse, Siena (6), basato su metodica EIA in monodeterminazione ed il sistema Euroline, Ditta Euroimmun AG, Luebeck, Germania, basato su metodica Western blot per la conferma della positività anti-HSV-1 ed anti-HSV-2 sia IgG che IgM.

In tutte le sedute analitiche sono stati testati dei sieri di controllo interno, positivo e negativo, della Ditta BioRAd, Milano, i cui risultati hanno soddisfatto i valori attesi con entrambi i sistemi di routine. Il laboratorio partecipa, inoltre, al programma DicoCARE VEQ Infettivologia, Programma di Valutazione Esterna di Qualità per Marcatori Sierologici di Malattie Infettive, prodotto dalla Ditta Care s.r.l. e distribuito dalla Ditta Medical System spa, Genova.

In Tabella 1 sono riportate la sensibilità e la specificità dichiarata, da ciascuna ditta, per ogni singolo reagente.

\section{RISULTATI}

I 182 campioni esaminati hanno presentato i seguenti risultati: per quanto riguarda il test HSV-1 IgG n. 139 (76.37 \%) sono risultati positivi e n. 43 (23.63\%) negativi con entrambi i sistemi, mostrando una concordanza del $100 \%$; per quanto riguarda i test HSV-1 IgM e HSV-2 IgG i 182 campioni sono risultati tutti negativi con entrambi i sistemi, presentando anche in questo caso una concordanza del $100 \%$. Solo per quanto riguarda il test HSV-2 IgM, 3 campioni sono risultati positivi con il sistema Analyzer I, mentre con il sistema RAD 120 tutti i campioni sono risultati negativi per HSV-1/2 IgM, presentando una discordanza dell'1.65 \%.

Nelle Tabelle 2, 3 e 4 sono riportati i risultati qualitativi, in valore assoluto ed in percentuale, derivanti dall'analisi dei campioni sui due sistemi utilizzati.

I 3 campioni risultati discordanti per il test HSV-2 IgM sono stati ulteriormente saggiati con i due sistemi in uso per la verifica dei risultati dubbi, ottenendo un risultato negativo sia con il sistema Chorus (metodo EIA) sia con il sistema Euroline (metodo Western blot).
Tabella 2. Risultati test HSV-I IgG

\begin{tabular}{lcc}
$\begin{array}{l}\text { Tabella } \\
\text { TEST } \\
\text { HSV-I IgG }\end{array}$ & ANALYZER I & RAD I 20 \\
\hline+ & $139(76.37 \%)$ & $139(76.37 \%)$ \\
\hline- & $43(23.63 \%)$ & $43(23.63 \%)$ \\
\hline Totale & 182 & 182 \\
\hline
\end{tabular}

Tabella 3. Risultati test HSV-I lgM e test HSV-2 $\lg G$ TEST

\begin{tabular}{lcc}
$\begin{array}{l}\text { HSV-I IgM } \\
\text { HSV-2 IgG }\end{array}$ & ANALYZER I & RAD I20 \\
\hline+ & 0 & 0 \\
\hline- & 182 & 182 \\
\hline Totale & 182 & 182 \\
\hline
\end{tabular}

Tabella 4. Risultati test HSV-2 IgM

\begin{tabular}{lcc}
\hline TEST & ANALYZER I & RAD I20 \\
HSV-2 IgM & $3(1.65 \%)$ & 0 \\
\hline+ & $179(98.35 \%)$ & 182 \\
\hline- & 182 & 182 \\
\hline Totale & &
\end{tabular}

\section{DISCUSSIONE E CONCLUSIONI}

L'analisi dei dati mette in evidenza come vi sia una completa sovrapposizione dei risultati ottenuti con i due sistemi per quanto riguarda la rilevazione degli anticorpi anti-HSV-1 IgG e IgM ed anti-HSV-2 IgG; per quanto attiene, invece, la ricerca degli anticorpi anti-HSV-2 IgM si rileva una lieve discordanza (1.65\%) soltanto su 3 campioni, che può essere valutata poco significativa vista la concordanza complessiva (98.35\%) dei dati comunque superiore al 95\%. È inoltre da considerare la negatività ottenuta con il test di conferma in Western blot sui campioni discordanti, che evidenzia la possibilità di incorrere in false positività utilizzando esclusivamente test di primo livello; d'altro canto i tre campioni discordanti, come è risultato da un'indagine retrospettiva, non provenivano da soggetti con sospetto di infezione da HSV.

I due sistemi, quindi, pur avvalendosi di tecnologie differenti, devono essere valutati in base alle esigenze organizzative ed al carico di lavoro del laboratorio; vista la sovrapponibilità dei risultati ottenuti, si può concludere che entrambi i sistemi possono essere utilizzati, con affidabilità, per la determinazione sierologica preliminare degli anticorpi anti-HSV.

\section{BIBLIOGRAFIA}

1. Brown ZA, Wald A, et al. Effect of sierologic status and cesarean delivery on trasmission rates of herpes simplex virus from mother to infant. JAMA 2003; 289: 203-9.

2. Gibbs RS, Amstey MS, Sweet RL, et al. Management of genital herpes infection in pregnancy. Obstet Gynecol, 1988; 71: 779-80.

3. Kimberlin DW. Neonatal Herpes Simplex Infection. Clin Micobiol Rev, 2004; 17 (1): 1-13.

4. Whitley RJ. Herpes simplex. In: Pickering LK. Red Book: report of the Committee on Infectious Diseases, 25th edition, Editors Elk Grove Village III: American Academy of Pediatrics. 2000; 309-18.

5. Whitley RJ. Neonatal herpes simplex virus infection. Curr Opin Infect Dis, 2004; 17: 243-6.

6. www.diesse.it

7. www.Euroimmun.it

8. www.radim.com

Tabella I. Sensibilità e specificità indicate dalle Ditte Euroimmun e Radim per i sistemi Analyzer I e RAD I20. ND: non dichiarata

\begin{tabular}{lccccc}
\hline & \multicolumn{2}{c}{ Sensibilità } & \multicolumn{2}{c}{ Specificità } \\
\cline { 2 - 6 } TEST & $\begin{array}{c}\text { Analyzer I } \\
\text { Euroimmun }\end{array}$ & $\begin{array}{c}\text { RAD I 20 } \\
\text { Radim }\end{array}$ & $\begin{array}{c}\text { Analyzer I } \\
\text { Euroimmun }\end{array}$ & $\begin{array}{c}\text { RAD I 20 } \\
\text { Radim }\end{array}$ \\
\hline HSV-I IgG & 100 & 99 & 100 & 95 \\
\hline HSV-2 IgG & 100 & 98 & 95.5 & 100 \\
\hline HSV-I IgM & 100 & - & 100 & - \\
\hline HSV-2 IgM & ND & - & 100 & - \\
\hline HSV-I/2 IgM & - & 94 & - & 98 \\
\hline
\end{tabular}

